- off a criminal charge when the death of two chimpanzees highlighted the facility's after-hours practice of leaving critically ill animals in the care of security guards with no veterinary training - a policy the NIH says has since been changed. This troubled history has led some to say that the Alamogordo colony has been through enough, and that it is time to let the chimps retire. "These animals have been used to an extensive degree," says John Gluck, an emeritus professor of psychology at the University of New Mexico in Albuquerque. "Is there a sense that we owe them something for that? I think we do."

Gluck says that when he first visited the Alamogordo colony in 1973, the stark environment and individual metal cages reminded him of a medieval prison. Since then, the buildings have been converted into a more comfortable habitat, complete with space for the chimpanzees to socialize and to exercise outside.

The 2000 US Chimpanzee Health Improvement, Maintenance, and Protection Act called for a system of sanctuaries for retired chimpanzees "no longer needed" for medical research. Only one such federal sanctuary has been built: Chimp Haven, near Keithville in Louisiana, which is home to some 100 retired chimps. But the government's contract with Chimp Haven expires in 2012 and there has been no discussion of extending it. At Alamogordo, the chimpanzees have enjoyed their unofficial retirement because Holloman Air Force Base, where they are located, dictates that they cannot be used for medical research on the premises.

But the NIH considers the Alamogordo centre a "research reserve" rather than a sanctuary. Closing the centre and moving the chimps to San Antonio will save US\$2 million a year, says Harold Watson, $\mathrm{NIH}$ programme director for chimpanzee management.

In Texas, the Alamogordo chimpanzees will join about 150 others in an environment much like that at their present facility, says Watson. They will be allowed to socialize and go outdoors unless specific medical protocols require isolation. "This colony has been together for a long time," he adds. “They'll be temporarily disrupted during the move, but then the original social groups can reform and that's the best way to do it." Most testing will involve little more than a few blood samples, and samples of liver tissue taken using very thin needles, he says.

If so, then sending the chimps back into the lab could be justifiable, says Ajit Varki, a biochemist at the University of California, San Diego. "We have the chimps in captivity," he says. "They cannot be returned to the wild. We should be able to do with chimps what we do with humans." -

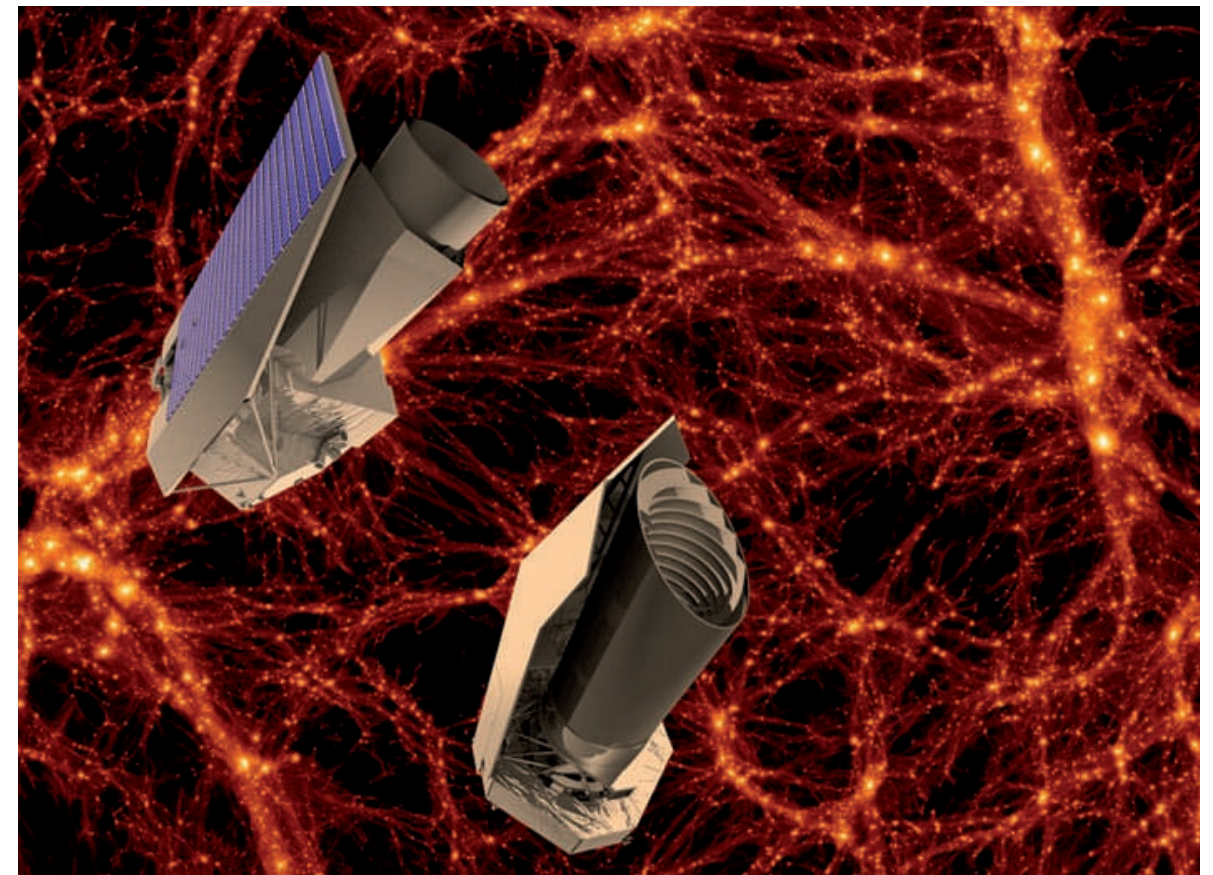

This depiction of two concepts for the Euclid observatory could foreshadow a real-world problem.

ASTRONOMY

\section{No scope for agency collaboration in space}

\section{BY ADAM MANN}

I ought to be a match made in heaven. Two telescopes - one European, one American - with similar research objectives and a combined price tag of more than US $\$ 2$ billion are both looking for support from funding agencies and the scientific community. In the current budget climate, it would seem natural for the projects to combine forces and push for one joint mission. Instead, it seems that in about a dozen years' time there will be two orbiting observatories doing the same job.

"Does it make sense to send two missions if their scientific goals are so similar?" asked James Kasting, a geoscientist from Pennsylvania State University in University Park, during a meeting of the 17-member NASA Advisory Council astrophysics subcommittee in Washington DC on 16-17 September. Other subcommittee members, who have the task of deciding how NASA should proceed on the telescopes, echoed the question. "Unless you change things, we might be doing the same thing twice," says chairman Alan Boss, an astrophysicist at the Carnegie
Institution for Science in Washington DC.

At stake is the chance to speed progress towards a desirable goal: a better understanding of dark energy, the mysterious phenomenon that has been proposed to explain the acceleration of the Universe's expansion. To investigate it, a telescope must be able to look for dark energy's subtle effect on the distribution of galaxies and dark matter, as well as on the motion of distant supernovae (see 'Illuminating dark energy') - ambitious requirements. Yet prospects for a partnership between NASA and the European Space Agency (ESA) to develop such a telescope have collapsed in a bureaucratic tangle.

Two options remain: NASA could become the minority partner in a Europe-led mission in exchange for ESA taking on a share of a similar US project, or they could proceed on their own separate but parallel tracks. Both options set up the possibility of a redundant effort.

The dual-mission dilemma was precipitated last month by the US National Academy of Sciences, in its release of the Astro2010 decadal survey, which sets goals and funding priorities for US astronomers and astrophysicists from 
2012 to 2021 . The highest-priority project in the queue is the James Webb Space Telescope (JWST), an infrared observatory now in testing and heading for launch in 2014. But once that is completed, the survey recommends that the United States develop a new mission, the WideField Infrared Survey Telescope (WFIRST).

Although described as a multi-purpose instrument, WFIRST is effectively a repackaging of the Joint Dark Energy Mission (JDEM), a shared NASA-Department of Energy (DOE) project to explore the nature of dark energy. And its objectives are strikingly similar to those of a proposed 1.2-metre European telescope called Euclid, in the works since 2007.

Last year, Jon Morse, director of NASA's astrophysics division, tried to broker a deal in which ESA would relinquish plans for Euclid and join the JDEM. The Europeans were interested, says Morse, but the deal fell apart when DOE officials complained that ESA would be providing the instruments that the DOE had planned to make. Then, with the JDEM bogged down (see Nature 461, 1182-1183; 2009), Morse met with ESA officials and offered to provide $20 \%$ of Euclid's funding and 2 astronomers for its 12-member science working group. But after the decadal survey was released and WFIRST emerged as a top priority, NASA was again looking to lead a combined mission. According to Ed Weiler, NASA's associate administrator for science, this time the response from ESA was "an unequivocal no", because Euclid was now too far along in its bid for a 2018 launch slot.

Stuck in line behind the JWST, it is unlikely that WFIRST will launch before 2022, which potentially relegates it to an also-ran in the quest to explore dark energy. So Morse and Weiler have proposed an arrangement that would see NASA contributing a third of Euclid's budget — around \$260 million over ten years — in exchange for an equivalent European commitment to WFIRST.

"When your budget is tight and you're trying to get high-priority science data this decade, this makes sense," said Morse.

But the quid-pro-quo deal is not so inviting to some on the panel, in part because the distinction between the missions is not compelling enough to require separate efforts. "One is a little better here and one is a little better there, but they seem to have a large overlap," said Vicky Kalogera, an astrophysicist at Northwestern University in Evanston, Illinois, and a

\section{TECHNIQUES}

\section{Illuminating dark energy}

To distinguish between competing ideas about the nature of dark energy, astronomers need to measure how it is affecting the expansion rate of the Universe. There are at least three ways to do this (below). The European Space Agency's proposed Euclid mission would use the first two, whereas NASA's Wide-Field Infrared Survey Telescope would do all three.

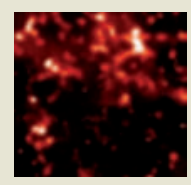

Baryon acoustic oscillations: in the early Universe, sound waves travelled through space, leaving behind patterns in the large-scale arrangement of baryons (protons and neutrons) in the Universe. By measuring the characteristic scale of these patterns, astronomers can use them as a cosmic ruler. Using this technique, researchers can infer how dark energy has stretched the Universe over time.

subcommittee member. Astronomers will gain little from WFIRST if it comes online a few years after Euclid. "Is it impossible to find a way to join with ESA and have a 50:50 'EuFIRST' mission?" Kalogera asked, a sentiment repeated several times during the discussion.

The problem,

"Is it impossible to find a way to join with ESA and have a 50:50 mission?" Morse said, is that to achieve all of the US decadal survey's goals, such a telescope would have to be bigger than the Europeans want; WFIRST is set to have a 1.5-metre aperture. "Euclid is competing for a medium-scale slot," said Fabio Favata, ESA coordinator for astronomy and fundamental physics missions. "We cannot do a telescope larger than 1.2 metres."

Euclid's mission proposal, which will be evaluated alongside two competing projects, is due on 29 October. Before then, the Euclid team must know how much, if anything, the United States wants to commit to it. ESA must decide by mid-2011 whether to advance Euclid or give the

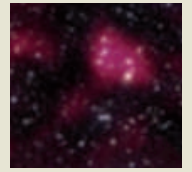

Weak gravitational lensing: extremely massive objects, such as galaxy clusters, bend light from objects behind them. The slight lensing of light from distant galaxies will help to reveal how dark matter is distributed in the foreground and how much influence dark energy has exerted on that space.

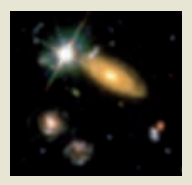

Supernovae distances: type la supernovae, which were once white dwarf stars, have consistent peak luminosities, so differences in their apparent brightness can be used to estimate the distance to far-away galaxies. Comparing the distances to the rate at which the galaxies are swept along by cosmic expansion gives cosmologists a better estimate of the Universe's expansion rate at different points in its history. A.M.

2018 launch slot to another mission.

Some subcommittee members say that a wasteful duplication could still be avoided. If Euclid is given the go-ahead, the mission particulars will come up for review, giving another chance for a joint US-European venture, says Boss. Even if the telescopes are not combined the final specification of both missions could change before either launches, says Arjun Dey, an astrophysicist at the US National Optical Astronomy Observatory in Tucson, Arizona.

At the end of the two-day meeting, the subcommittee was split between increasing involvement in Euclid and dropping it entirely. For now, they have chosen to return to the $20 \%$ funding proposal and reconvene in late October to decide on further action.

"This is a big decision that will affect many people, and many on the panel were anxious," says Boss. "In an ideal world, we would sit down and start from scratch." When asked about fears that a two-mission scenario could become a public-relations nightmare, he said, "If these concerns don't get solved, that might become a big problem."

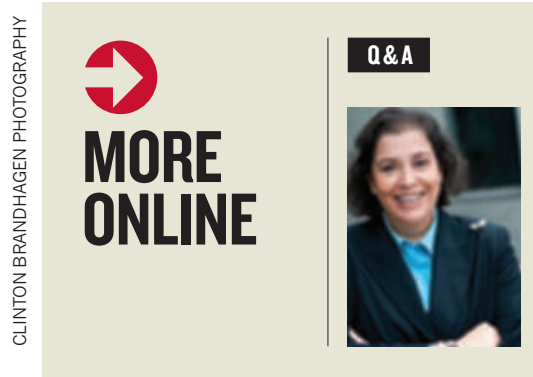

- Gabriela Chavarria, top science adviser to the US Fish and Wildlife Service, on the agency's scientific direction. go.nature.com/pjXCsl

\section{ON THE NEWS BLOG}

- Obama's green team in hiring probe go.nature.com/6rjFn4

- Can science help the Millennium

Development Goals succeed? go.nature.com/2CkGmt

- Game over for British science? go.nature.com/rl2Crt

\section{PICTURE STORY}

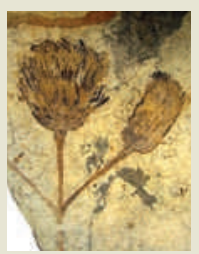

Rare fossil reveals the origins of daisies and sunflowers. go.nature.com/owWRqU 